\title{
Preventing Obesity in 2-5-Year Olds: A Pathway to Advancing Intervention Research
}

\author{
Christina D. Economos, $\mathrm{PhD}^{1,2}$ and Debra Haire-Joshu, $\mathrm{PhD}^{3}$
}

I $\mathrm{n}$ this issue, the comprehensive Childhood Obesity Evidence Base Project: A Systematic Review and Meta-Analysis Piloting a Taxonomy of Intervention Components to Prevent Obesity in Children 2 to 5 Years of Age, 2005 to 2019 is presented through four articles describing the methods, taxonomy, rationale, and results. ${ }^{1-4}$ We are encouraged by the key findings of this ambitious project regarding the effectiveness of obesity prevention strategies targeting young children over the past 15 years, but we also believe it revealed shortcomings, including limited application, inconsistent measurement tools, failure to sample adequate children of low socioeconomic status, and modest impact. We discuss several research design, methods, and implementation areas that need to be improved if we expect increased and far-reaching efficacy for these interventions. We also maintain that due to the time lag between conducting interventions and publishing results, the available evidence is somewhat out of sync with current scientific thinking and up-to-date societal needs.

This taxonomic meta-analysis by Scott-Sheldon et al. summarized the evidence that exists for early childhood obesity interventions and concluded that they are effective in reducing BMI. ${ }^{5}$ Included were 51 studies evaluating 58 interventions. Analyses indicated that children in the intervention group had a smaller BMI increase at the immediate post-test $(g=0.10,95 \% \mathrm{CI}=0.02-0.18 ; k=55$; 0 weeks $)$ and at the last follow-up $(g=0.19,95 \% \mathrm{CI}=0.02-$ $0.37 ; k=14$; range $=18-143$ weeks) relative to the comparison group. When pooling the separate BMI metrics, analyses showed that children in the intervention group had a lower BMI percentile at the immediate post-test $(g=0.17$, $95 \% \mathrm{CI}=0.01-0.32 ; k=19$ and last follow-up $(g=0.60$, $95 \% \mathrm{CI}=0.10-1.11 ; k=6)$ and a lower BMI z-score at last follow-up $(g=0.23,95 \% \mathrm{CI}=0.03-0.42 ; k=12)$ relative to the comparison group. Intervention children also had a lower weight at the immediate post-test $(g=0.39,95 \%$ $\mathrm{CI}=0.15-0.62 ; k=16)$ and last follow-up $(g=0.31,95 \%$ $\mathrm{CI}=0.05-0.58 ; k=11)$ assessment compared with the comparison group. Three intervention components moderated efficacy: engaging caregivers in praise/encouragement for positive health-related behavior, providing education about the importance of screen time reduction to caregivers, and engaging pediatricians/health care providers. Interventions that sampled fewer children of low socioeconomic status $(\beta=-0.20, p=0.002)$, recruited samples from clinical practices $(\beta=0.20, p<0.001 ;$ e.g., pediatrician offices, primary care), and implemented the intervention in a clinical practice $(\beta=0.24, p<0.001)$ were more likely to reduce children's BMI at the immediate post-test.

Although there was consistency across successful intervention components, many of the studies reviewed used single-strategy interventions (curricula, written resources, education, and limited training) to create behavior change at the child/caregiver level. In real-world settings, however, implementing these components in adequate measures will be challenging. Furthermore, the literature is calling for multilevel multicomponent interventions, with a health equity focus, using systems and implementation science to shape and guide the work. We must shorten our learning-to-implementation cycles to prevent interventions from being out-of-pace with current scientific thinking. This commentary highlights several areas of study that need to be addressed if we are to advance research toward new knowledge and solutions. Another commentary reflects on the importance and potential implications of the project from a methodological perspective. ${ }^{5}$

A health equity lens should drive childhood obesity research. Inequities in the social determinants of health are

'ChildObesityl80, Boston, MA, USA.

${ }^{2}$ Division of Nutrition Interventions, Communication, and Behavior Change, Friedman School of Nutrition Science and Policy, Tufts University, Boston, MA, USA.

${ }^{3}$ Center for Diabetes Translation Research, Center for Obesity Prevention and Policy Research, Brown School at Washington University, St. Louis, MO, USA. 
complex and deeply rooted in systemic structures, discrimination, and biases that are underlying drivers of childhood obesity prevention and treatment. ${ }^{6}$ Yet this National Collaborative on Childhood Obesity Research (NCCOR) review found the majority of research focused on individually oriented interventions, in which the child/parent must change, adapt, or overcome their context to succeed. We should recognize these inequities as drivers of obesity and apply a health equity lens to future research, identifying social determinants as targets of studies needed to understand and "move the needle" on eliminating childhood obesity.

Community-engaged recruitment, retention, and implementation strategies can guard against differential treatment effects. We need to investigate why some children are enrolled and remain in studies and others do not. Research with children and families from underrepresented backgrounds (African Americans, blacks, Hispanics, Latinx, Native Americans, and American Indians) and those with socioeconomic disadvantage remains inadequate in the United States. ${ }^{7,8}$ Intentional robust strategies to engage these children and their families in valuable health research may contribute to eliminating health disparities, shaping targeted policies, allocating resources appropriately, and building equity across communities.

Research must now move toward upstream determinants of marginalized communities, settings, and populations as a focus of multilevel interventions. A critical limitation of interventions targeting obesity and health disparities is that they have tended to focus on single- or individual-level factors. Although interventions targeting family-level change can be effective, they have limited reach and require a number of components, including deep and sustained engagement, motivation to overcome barriers, and reconstruction of the environment. ${ }^{9,10}$ Multilevel interventions that target the broader community, societal, and environmental contexts that affect individual behavior or risk produce greater reductions in health disparities than do approaches targeting a single level. ${ }^{11}$ Research that clarifies the pathways to implementation of interventions and generates new knowledge is necessary if we intend to ensure policies surrounding obesity prevention and treatment provide equal benefit to all populations. There are challenges in delivering interventions across several levels, but the necessity of employing a multilevel approach to address childhood obesity is coming into wider acceptance.

Childhood obesity research should prioritize the broad reach of interventions. As noted by the NCCOR review, vulnerable populations are underrepresented across childhood obesity studies. This may yield "one size fits all" interventions that can have variable results on subpopulations impacted by underlying contextual inequities. ${ }^{12}$ This inherently limits the external validity of the findings and can contribute to disparities in childhood obesity. Central to a focus on equity is the representation in enrollment and engagement of vulnerable populations as engaged participants. Partnerships or stakeholder involvement in developing or testing interventions among vulnerable populations within context is critical to assure implementation strategies and outcomes that are equitable for all.

Rigorous and consistent reporting of implementation strategies and outcomes need to be advanced. Currently, there is wide variation in reporting of obesity intervention components; this makes evaluation and comparison across studies challenging. ${ }^{13}$ For example, this review found limited or a lack of consistent information on descriptions of interventions, including how they were adapted, their reach, extent of stakeholder involvement, the role of context, or other elements that might influence equitable uptake. Systematic reporting to inform methods that enhance the adoption, implementation, and sustainability of interventions will allow for comparisons across studies and groups. Consistent with a focus on health equity, rigorous and consistent reporting of intervention elements are needed to ensure adequate assessment impact through an equity lens.

Implementation science offers a systematic structure to inform methods that enhance the adoption, implementation, and sustainability of interventions, and will allow for systematic comparisons across populations. It allows for the integration of evidence-based interventions across various contexts, taking into account many factors, including characteristics of users, types of evidence needed, organizational climate, and culture. Implementation science can address inequities through the study of factors, processes, and strategies at multiple levels (e.g., child/ family, provider, organizations, and communities) and across systems of care that influence the uptake, use, and ultimately the sustainability of effective interventions and policies in community settings. ${ }^{14}$

Systems science should be used to deal with complexity in design, implementation, and analyses. Increasing adoption of complex systems methodologies to model, measure, and engage will help to manage the complexity that comprehensive interventions present. ${ }^{15}$ Community-level research with stakeholder engagement can benefit from participatory and group model-building activities that produce systems mapping and causal loop diagrams, enabling a visualization of how relationships between community elements and processes interrelate and can illustrate leverage points. ${ }^{16}$ Systems modeling and other simulation methods can help capture the complex dynamics involved in successful and sustainable implementation of interventions, uncovering key findings beyond traditional analyses.

If meaningful and widespread progress in obesity prevention is the goal, we look to scientists, practitioners, funders, and policy makers to consider these areas in the next generation of intervention research.

\section{References}

1. King H, Magnus M, Hedges LV, et al. Childhood Obesity Evidence Base Project: Methods for taxonomy development for application in taxonomic meta-analysis. Childhood Obes 2020;16: S2-7-S2-20. 
2. Hedges LV, Saul JD, Cyr C, et al. Childhood Obesity Evidence Base Project: A rationale for taxonomic vs conventional metaanalysis. Childhood Obes 2020;16:S2-1-S2-6.

3. Young-Hyman D, Kettel Khan L. Childhood Obesity Evidence Base Project. Building translational capacity through meta-analytic methods. Childhood Obes 2020;16:S2-49-S2-54.

4. Scott-Sheldon LAJ, Hedges LV, Cyr C, et al. Childhood Obesity Evidence Base Project. A systematic review and meta-analysis of new taxonomy of intervention components to improve weight status in children 2-5 years of age, 2005-2019. Childhood Obes 2020;16: S2-21-S2-48.

5. Kumanyika SK. Learning more from what we already know about childhood obesity prevention. Childhood Obes 2020;16: S2-55-S2-58.

6. Kumanyika SK. A framework for increasing equity impact in obesity prevention. Am J Public Health 2019;109:1350-1357.

7. Yancey AK, Ortega AN, Kumanyika SK. Effective recruitment and retention of minority research participants. Annu Rev Public Health 2006;27:1-28.

8. Cui Z, Seburg EM, Sherwood NE, et al. Recruitment and retention in obesity prevention and treatment trials targeting minority or low-income children: A review of the clinical trials registration database. Trials 2015;16:564.

9. Ash T, Agaronov A, Young T, et al. Family-based childhood obesity prevention interventions: A systematic review and quantitative content analysis. Int J Behav Nutr Phys Act 2017;14:113.

10. Enright G, Allman-Farinelli M, Redfern J. Effectiveness of familybased behavior change interventions on obesity-related behavior change in children: A realist synthesis. Int $J$ Environ Res Public Health 2020;17:4099.

11. Institute of Medicine. Accelerating Progress in Obesity Prevention: Solving the Weight of the Nation. Washington, DC: The National Academies Press, 2012.
12. Baumann AA, Cabassa LJ. Reframing implementation science to address inequities in healthcare delivery. BMC Health Serv Res 2020;20:190.

13. Brown AW, Altman DG, Baranowski T, et al. Childhood obesity intervention studies: A narrative review and guide for investigators, authors, editors, reviewers, journalists, and readers to guard against exaggerated effectiveness claims. Obes Rev 2019;20:15231541.

14. Estabrooks PA, Brownson RC, Pronk NP. Dissemination and implementation science for public health professionals: An overview and call to action. Prev Chronic Dis 2018;15:E162.

15. Hammond RA. Complex systems modeling for obesity research. Prev Chronic Dis 2009;6:A97.

16. Hennessy E, Economos CD, Hammond RA; SUS Systems Map Team and the COMPACT Team. Integrating complex systems methods to advance obesity prevention intervention research. Health Educ Behav 2020;47:213-223.

Address correspondence to:

Christina D. Economos, PhD

Division of Nutrition Interventions, Communication, and Behavior Change

Friedman School of Nutrition Science and Policy

Tufts University

150 Harrison Avenue

Boston, MA 02111

USA

E-mail: christina.economos@tufts.edu 